\title{
ULTRASONIC ATTENUATION DUE TO RESONANT INTERACTION WITH A DISTRIBUTION OF LEVEL SPLITTINGS OF THE GROUND STATE OF SHALLOW ACCEPTORS IN Ge
}

\author{
E. Ortlieb, Hp. Schad and K. Lassmann \\ Max-Planck-Institut für Festkörperforschung, Heilbronnerstrasse 69, 7000 Stuttgart 1, Deutschland
}

(Received 22 March 1976 by M. Cardona)

\begin{abstract}
The ultrasonic attenuation in $\mathrm{Ge}(\mathrm{Ga}, \mathrm{In})$ has been measured in the frequency range from $500 \mathrm{MHz}$ to $2.5 \mathrm{GHz}$, and from room temperature down to $1 \mathrm{~K}$. Below $10 \mathrm{~K}$ the attenuation rises as $\omega^{2} / T$. For the first time saturation of the attenuation has been observed for ground state of a shallow acceptor. These results can be interpreted as due to resonance interaction with level splittings of a broad distribution with width of about $0.1 \mathrm{meV}$.
\end{abstract}

IT IS WELL-KNOWN that the fourfold degenerate $\Gamma_{8}$ ground state of shallow acceptors in cubic semiconductors may be partly split due to random local fields. In $\mathrm{Ge}$ there is some evidence from magnetothermal and piezothermal conductivity measurements ${ }^{1,2}$ that the distribution of such splittings may be rather wide even in clean crystals of low doping and dislocation density. Such a distribution $N(\delta)$ of level splittings $\delta$ may be probed to some extent by resonant interaction $(\hbar \omega=\delta)$ of an ultrasonic wave at various frequencies.

We have, therefore, investigated the resonance attenuation of longitudinal sound waves in several $p$-type $\mathrm{Ge}$ crystals (see Table 1) between $500 \mathrm{MHz}$ and $2.5 \mathrm{GHz}$. CdS transducers were evaporated onto the samples.* The attenuation was measured by the pulse-echo method utilizing a coaxial cavity which is tunable in a broad frequency band by means of a capacitive plunger. ${ }^{3}$ The intensity dependent part of the attenuation was determined in such a way as to eliminate non-linearities of the receiver by working at the same level with a calibrated attenuator at the receiver input. The highest intensity at $1 \mathrm{GHz}$ was estimated to be of the order of $1 \mathrm{~mW} / \mathrm{cm}^{2}$.

Apart from some quantitaiive differences we obtained analogous results for the four crystals: below about $50 \mathrm{~K}$ down to $20 \mathrm{~K}$ the attenuation first falls $\propto T^{4}$ as expected if phonon-phonon interaction is dominant. The interesting region in this context begins below $10 \mathrm{~K}$. There the at tenuation rises again $\propto 1 / T$ as shown in Fig. 1 for several frequencies. Since the absolute value of the attenuation in this region is obscured due to nonexponential decay of the echo train because of temperature independent geometrical effects we subtract the apparent residual attenuation at $1 / T=0$ and obtain the curves shown in Fig. 2. Here we have plotted $\Delta \alpha$ vs

\footnotetext{
* We are indepted to W. Arnold, Max-Planck Institut, Grenoble, for the transducers.
}

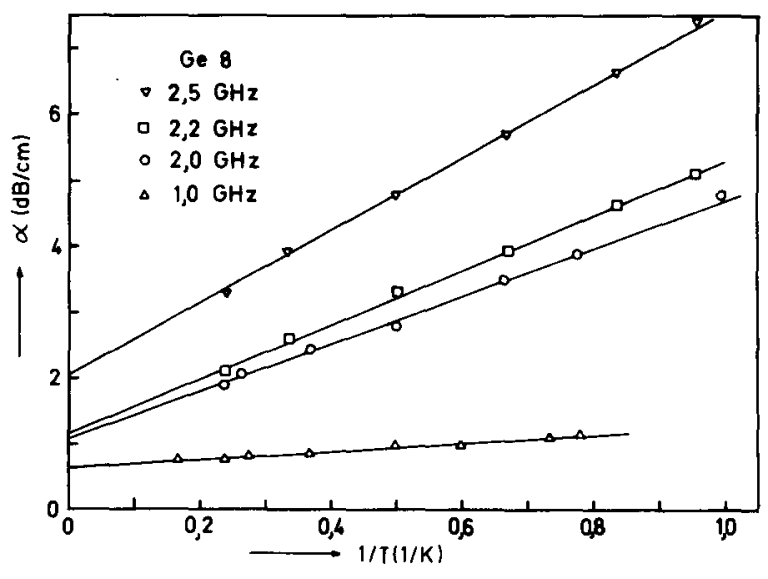

Fig. 1. Dependence of the attenuation on $1 / T$ for low intensities.

frequency squared and get straight lines within our accuracy of some $10 \%$. Thus, below $20 \mathrm{~K}$ the attenuation rises as $\omega^{2} / T$. This is observed for all crystals if the acoustic intensity is low enough. At higher intensities we find in all cases an amplitude dependent attenuation as shown in Fig. 3 for two examples. Such a behavior is typical for resonant interaction with an anharmonic system. For an evaluation of the measurements we apply formulae analogous to those developed for the acoustic attenuation in the distributed-two-level-model for glasses. ${ }^{4}$ We have discussed these formulae to some extent in reference 5 . If the distribution $N(\delta)$ does not vary much within the linewidth of the resonance one obtains for the resonart part of the acoustic attenuation $\alpha_{\text {res }}$ at low acoustic intensities

$$
\alpha_{\text {res }}=\left(h / 4 \rho v^{3} k_{B}\right) D^{2} N(\delta=\hbar \omega) \cdot\left(\omega^{2} / T\right)
$$

with 
Table 1. Sample parameters and widths of distribution $\delta_{0}$ (Ge 10 has been kindly supplied by Haseler ${ }^{2}$ )

\begin{tabular}{lllll}
\hline Crystal & Ge 1 & Ge 8 & Ge 9 & Ge 10 \\
Orientation & {$[111]$} & {$[111]$} & {$[111]$} & {$[110]$} \\
Dopant & $\mathrm{Ga}$ & In & In & In \\
Concentration $\left(10^{15} \mathrm{~cm}^{-3}\right)$ & 30 & 7 & 20 & 8 \\
Dislocation density $\left(\mathrm{cm}^{-2}\right)$ & 700 & 1500 & 0 & 300 \\
$\delta_{0}(\mathrm{meV})$ & 0.08 & 0.11 & 0.06 & 0.07 \\
\hline
\end{tabular}

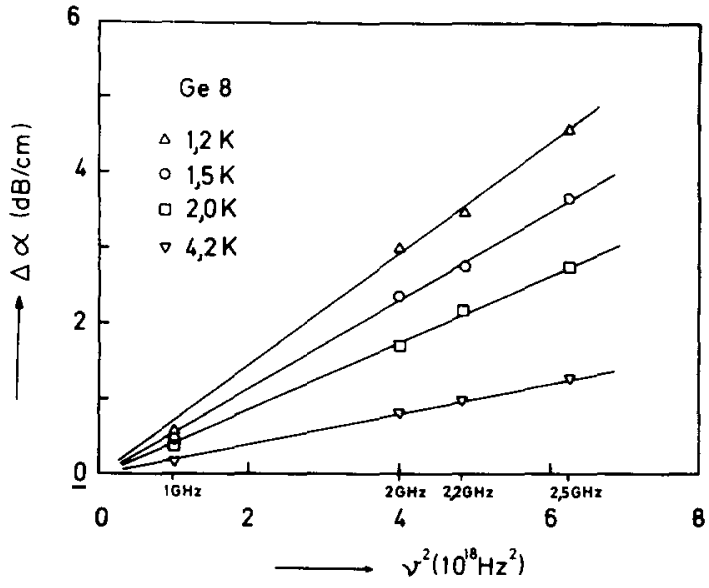

Fig. 2. Dependence of the attenuation on $\nu^{2}$ at several temperatures (apparent residual attenuation subtracted).

$$
\int_{-\infty}^{+\infty} N(\delta) \mathrm{d} \delta=N .
$$

Here, $N$ is the concentration of neutral acceptors, $\rho$ the density of the crystal, $v$ the sound velocity. $D$ is an appropriate combination of the two deformation potential constants of the acceptor describing an averaged interaction strength of the ultrasonic wave with the random splittings in resonance. $D$ is difficult to estimate without specific knowledge of the splitting distribution. It should be the same combination under analogous experimental conditions. If one knew $D$ one would obtain the absolute value of $N(\delta)$ from the absolute value of $\alpha$, and, since $D$ is frequency-independent at ultrasonic frequencies, its variation with $\delta=\hbar \omega$ in the range measured. Further information may be obtained from the intensity-dependent part of the resonant attenuation due to the saturation of the levels on speaking terms at high acoustic intensities. From the critical intensity one can estimate the relaxation time $\tau$ of the levels. $4,6-8$ Relaxation attenuation, which is not saturable, may be also of importance. ${ }^{9}$ Here we have to sum over all contributions ${ }^{5} \delta \gg \hbar \omega$

$$
\alpha(\delta)=N(\delta) f(\delta, T) \omega^{2} \tau(\delta, T) /\left\{1+[\omega \tau(\delta, T)]^{2}\right\}
$$

where $f(\delta, T)$ is the relaxation strength and $\tau(\delta, T)$ the

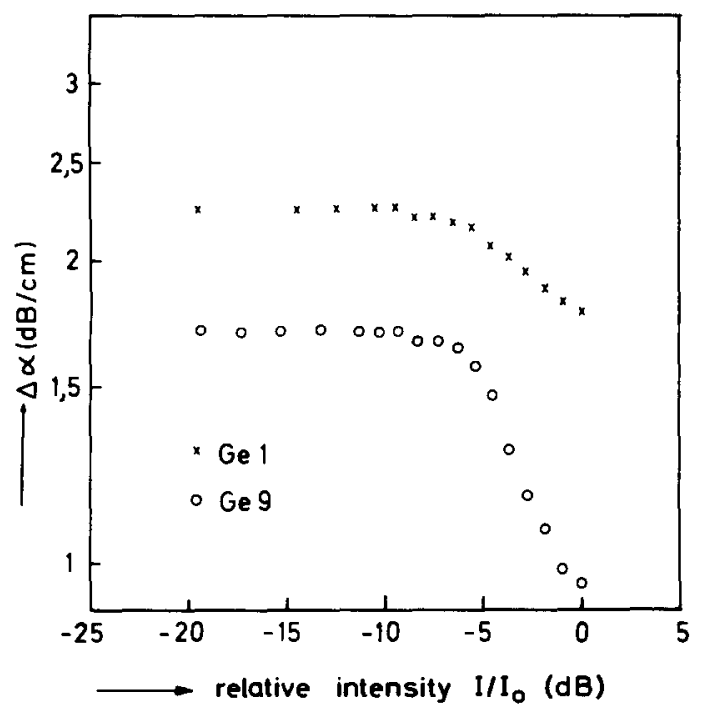

Fig. 3. Power dependent part of the attenuation for two crystals at $1 \mathrm{GHz}$.

relaxation time. The latter may be due to Raman, Arrhenius-Orbach, or direct relaxation processes of the levels of an individual impurity. None of these processes will lead to an $\omega^{2} / T$ dependency of the relaxation attenuation. At higher concentrations relaxation via interaction of impurities may be significant.

Our results can be interpreted as follows. The $\omega^{2} / T$ rise of the attenuation is due to resonant interaction. The distribution $N(\delta)$ is constant in the range measured, that is between 4 and $10 \mu \mathrm{eV}$. For a rough estimate of the width of the distribution from the absolute value of the attenuation we may assume $N(\delta)$ to be constant up to a maximum value $\delta_{0}$. The values $\delta_{0}$ for the different crystals obtained with $D=(2 / 3) D_{u}^{a / 10,11}$ are listed in Table 1 . They are consistently rather large, but may be smaller if $N(\delta)$ is peaked at some non-zero value or if $D$ has to be reduced. For a rectangular distribution up to $0.1 \mathrm{meV}$ relaxation at tenuation $\alpha_{\text {rel }}$ should be negligible. A relaxation peak due to two-phonon (Raman) processes is estimated to occur near $20 \mathrm{~K}$. However, it would be too small $(\sim 0.2 \mathrm{~dB} / \mathrm{cm})$ and too broad to be discernible from the resonant and phonon-phonon interaction contributions to the attenuation. From the estimated critical 
intensity $J_{c} \sim 0.2 \mathrm{~mW} / \mathrm{cm}^{2}$ we deduce $\tau \sim 20 \mathrm{nsec}$ at $2 \mathrm{GHz}, 1 \mathrm{~K}$ which is smaller by two orders of magnitude than the value calculated for direct relaxation. The short relaxation time may indicate impurity interaction but in view of the uncertainties of $J_{c}$ and $D$, a more detailed and careful investigation of the saturation is necessary.

As can be seen from Table 1 , the values of $\delta_{0}$ do not vary significantly with acceptor concentration or dislocation density. The value of $\delta_{0}$ we deduce from our measurements is in accordance with that derived from piezothermal conductivity measurements ${ }^{2}$ but it is much larger than found in ultra high purity crystals of low acceptor concentration by an optical method. ${ }^{12}$ Inter- action between acceptors ${ }^{13}$ cannot be ruled out for our dopings, however, one would expect a strong dependence on concentration. Jahn-Teller effect of the acceptor ground state has been discussed, ${ }^{1}$ but from the stability criterion given by $\mathrm{Bir}^{14}$ a JTE should not be stable for these shallow acceptors. Splittings $\delta$ due to electric fields from ionized impurities should be small compared to $\delta_{0}$ for any compensation ratio at our dopings $;^{15}$ and the same applies to the strain fields associated with the dislocation densities of our crystals. Another possibility would be a large concentration of an electrically inactive impurity, such as hydrogen, oxygen, or carbon, being responsible for the internal fields.

\section{REFERENCES}

1. CHALliS L.J. \& HALBO L., Phys. Rev. Lett. 28, 816 (1972).

2. CHALLIS L.J., HASELER S.C., PARSONS M.W.S. \& RIVALLIN J., Proc. 2nd Int. Conf. on Phonon Scattering in Solids, Nottingham (England). Plenum Press New York (in press).

3. KINDER H., Thesis, Göttingen (1968).

4. JÄCKLE J., Z. Phys. 267, 212 (1972).

5. LASSMANN K. \& SCHAD Hp., Solid State Commun. 18, 449 (1976).

6. FLETCHER J.R., J. Phys. C3, 1349 (1970).

7. ARNOLD W., HUNKLINGER S., STEIN S. \& DRANSFELD K., J. Non-Cryst. Sol. 14, 192 (1974).

8. JOFFRIN J. \& LEVELUT A., J. de Phys. 36, 811 (1975).

9. SCHAD Hp. \& LASSMANN K., Phys. Lett. $A$ (in press).

10. FJELDLY T., ISHIGURO T. \& ELBAUM C., Phys. Rev. B7, 1392 (1972).

11. JONES R.L. \& FISHER P., Phys. Rev. B2, 2016 (1970).

12. HALLER E.E. \& HANSEN W.L., Solid State Commun. 15, 687 (1974).

13. SUZUKI K. \& MIKOSHIBA N., Proc. Conf. on Phonon Scattering in Solids, Paris, 1972, p. 125. La Documentation Française, Paris (1972).

14. BIR G.L., Sov. Phys. JETP 24, 372 (1967).

15. BIR G.L., BUTIKOV E.J. \& PIKUS G.E., J. Phys. Chem. Solids 24, 1467 (1962). 